\title{
EFFECT OF INTEGRATED NUTRIENT MANAGEMENT ON NUTRIENT UPTAKE AND SUSTAINABLE GRAIN YIELD IN TRANSPLANTED AMAN RICE
}

\author{
M.S. Naher ${ }^{1^{*}}$ and A.K. Paul ${ }^{2}$ \\ ${ }^{1}$ Spices Research Centre, Shibganj, Bogra, Bangladesh \\ ${ }^{2}$ Sher-e-Bangla Agricultural University, Dhaka, Bangladesh
}

\begin{abstract}
A field experiment was carried out at Sher-e-Bangla Agricultural University (SAU) Farm during July to November 2011 to evaluate the effect of integrated nutrient management (INM) on T. Aman rice (cv. BRRI dhan40). The field belongs to Red Brown Terrace soil under Tejgoan series having silt loam texture and $6.0 \mathrm{pH}$. Application of $70 \%$ NPKS fertilizers $+4 \mathrm{t} \mathrm{ha}^{-1}$ dhaincha (Sesbania rostrata) $\left(\mathrm{T}_{1}\right)$ produced the highest grain yield $\left(5.90 \mathrm{t} \mathrm{ha}^{-1}\right)$, the second highest yield $\left(5.85 \mathrm{t} \mathrm{ha}^{-1}\right)$ was obtained from $80 \%$ NPKS $+2 \mathrm{t} \mathrm{ha}^{-1}$ dhaincha $\left(\mathrm{T}_{2}\right)$ treatment. The grain yield increased by 31.2 to $86.3 \%$ over control depending on the treatments. Treatments $\mathrm{T}_{1}$ and $\mathrm{T}_{2}$ resulted in higher $\mathrm{N}, \mathrm{P}, \mathrm{K}$ and $\mathrm{S}$ uptake by rice. The N, P, K and $\mathrm{S}$ concentrations of rice grain was higher for the treatments where Sesbania and cow dung were together applied in combination with NPKS fertilizers. The combined application of chemical fertilizers and organic manure increased organic carbon (OC), total $\mathrm{N}$, available $\mathrm{P}, \mathrm{K}$ and $\mathrm{S}$ contents in post-harvest soil. The overall results indicate that the integrated use of chemical fertilizer and organic manure can help increase grain yield of rice without deteriorating soil fertility.
\end{abstract}

Keywords: Integrated nutrient management, rice yield, soil fertility

\section{INTRODUCTION}

Agriculture in Bangladesh is predominately rice based and Bangladesh is the fourth rice (Oryza sativa) producing country in the world (BRRI, 2006). It is grown in 28.06 million hectares of land in which T. aman (rainfed wet season rice) covers about 13.99 million hectares and produces about 12.21 million metric tons of rice (BBS, 2010) by utilizing varied soil fertility levels and fertilizer management. Soil fertility deterioration is a major constraint for higher rice yield in Bangladesh. The increasing land use intensity without adequate and balanced use of chemical fertilizers and with little or no use of organic manures have caused severe fertility

\footnotetext{
"Corresponding author email: mahmud.nahar@yahoo.com
} 
deterioration of our soils resulting in stagnating or even declining of crop productivity. The farmers of this country use, on an average, $172 \mathrm{~kg}$ nutrients/ha (follow similar style) annually (132 kg N $+27 \mathrm{~kg} \mathrm{P}+17 \mathrm{~kg} \mathrm{~K}+4 \mathrm{~kg} \mathrm{~S}$, and $2 \mathrm{~kg} \mathrm{Zn}$ ), while the crop removal is about $250 \mathrm{~kg} \mathrm{ha}^{-1}$ (Islam, 1995). Since fertile soil is the fundamental resource for higher crop production, its maintenance is a prerequisite for long-term sustainable crop productivity. Soil organic matter is a key factor for sustainable soil fertility and crop productivity. A good soil in Bangladesh should have an organic matter content of at least $2.5 \%$ (BARC, 2012). According to an appraisal report of Bangladesh soil resources, soils of about $6.10 \mathrm{~m}$ ha contain very low (less than 1\%) organic matter, $2.15 \mathrm{~m}$ ha contain low (1-2\%) organic matter and the remaining $0.90 \mathrm{~m}$ ha contain more than $2 \%$ organic matter (Mondal, 2000). Green manure prepared from Sesbania spp. is a cheaper and feasible alternative to other organic sources. Green manuring species such as Sesbania rostrata can fix sufficient amounts of $\mathrm{N}$ for optimum rice yield without any inorganic $\mathrm{N}$ input (Meelu et al., 1992). Soil fertility declining day by day is a major reason for lower crop yield in Bangladesh. Intensive cropping, use of modern varieties, use of higher doses of nitrogenous fertilizers with little or no addition of organic manure are the principal factors for soil fertility depletion in the country. A crop production system with high yield targets cannot be sustainable unless balanced nutrient inputs are supplied to soil against nutrient removal by crops (Bhuiyan et al., 1991). Available data indicate that the soil fertility in Bangladesh is in declining trend (Karim et al., 1994; Ali et al., 1997) which is responsible for declining crop yields (Cassman et al., 1995). Neither organic manure nor chemical fertilizer alone can increase satisfactory yield under intensive farming.

Organic fertilizers has positive effect on root growth by improving the root rizosfere conditions (structure, humidity, etc.) and also plant growth is encouraged by increasing the population of microorganisms (Shaheen et al., 2007). Organic fertilizers contain plant nutrients. Organic acids which occur in decomposition increases the benefits of nutrients (Anonymous, 2010). Organic sources of nutrients applied to preceding crop can benefit the succeeding crop to a great extent (Hedge, 1998) through integrated use of organic and inorganic sources of nutrients (Singh et al., 2001). Nambiar (1997) viewed that integrated use of organic manure and chemical fertilizers would be quite promising not only in providing greater stability in production, but also in maintaining better soil fertility. Based on the soil fertility problem as discussed above, the present study was undertaken to investigate the effect of combined use of chemical fertilizers and organic manures in T. aman (BRRI dhan40) production.

\section{MATERIALS AND METHODS}

The experiment was conducted in the experimental field of Sher-e-Bangla Agricultural University, Dhaka, Bangladesh during July to November 2011. The experiment was laid out in a randomized complete block design (RCBD), with three 
replications. Each block was divided into eight unit plots as treatments with raised bunds around. The unit plot size was $3 \mathrm{~m} \times 2 \mathrm{~m}\left(6 \mathrm{~m}^{2}\right)$. The treatment combinations used were $\mathrm{T}_{0}$ (Control, no fertilizer), $\mathrm{T}_{1}\left(\mathrm{~N}_{120} \mathrm{P}_{15} \mathrm{~K}_{45} \mathrm{~S}_{20} \mathrm{~kg} \mathrm{ha}^{-1}\right.$, recommended dose), $\mathrm{T}_{2}\left(80 \% \mathrm{NPKS}+2 \mathrm{tha}^{-1} \mathrm{DH}\right), \mathrm{T}_{3}\left(80 \% \mathrm{NPKS}+4 \mathrm{tha}^{-1} \mathrm{CD}\right), \mathrm{T}_{4}(80 \% \mathrm{NPKS}+1 \mathrm{t}$ $\left.\mathrm{ha}^{-1} \mathrm{DH}+2 \mathrm{tha}^{-1} \mathrm{CD}\right), \mathrm{T}_{5}\left(70 \% \mathrm{NPKS}+4 \mathrm{tha}^{-1} \mathrm{DH}\right), \mathrm{T}_{6}\left(70 \% \mathrm{NPKS}+8 \mathrm{tha}^{-1} \mathrm{CD}\right)$, $\mathrm{T}_{7}\left(70 \% \mathrm{NPKS}+2 \mathrm{tha}^{-1} \mathrm{DH}+4 \mathrm{tha}^{-1} \mathrm{CD}\right), \mathrm{T}_{8}\left(50 \% \mathrm{NPKS}+6 \mathrm{tha}^{-1} \mathrm{DH}\right), \mathrm{T}_{9}(50 \%$ NPKS $\left.+12 \mathrm{tha}^{-1} \mathrm{CD}\right), \mathrm{T}_{10}\left(50 \% \mathrm{NPKS}+3 \mathrm{tha}^{-1} \mathrm{DH}+6 \mathrm{t} \mathrm{ha}^{-1} \mathrm{CD}\right)$ and assigned in a randomized complete block design with three replications. The unit plot size was 3$\mathrm{x} 2-\mathrm{m} \times 2.0 \mathrm{~m}$. Full amount of $\mathrm{P}$ as TSP, $\mathrm{K}$ as MP and $\mathrm{S}$ as gypsum were applied during final land preparation. Urea was applied in 3 equal splits: one third at basal, one third at 30 days after transplanting (DAT) and the remaining one third was applied at 55 DAT. Two different types of organic manure viz., dhaincha (Sesbania rostrata) and cow dung (CD) were used. Dhaincha was applied before 2 weeks and CD was applied before one week of transplanting. Forty days old seedlings of BRRI dhan 40 were uprooted from seedbed and transplanted on 14 August 2011 at a spacing of $20 \mathrm{~cm}$ x $20 \mathrm{~cm}$ using 3 seedlings hill $^{-1}$. The crop was harvested when $80-90 \%$ of the grains were turned into straw colored on 26 November 2011. The plant height was measured from the ground level to the top of the panicle. From each plot, plants of 10 hills were measured and averaged. The measurement of panicle length was taken from basal node of the rachis to the apex of panicle. Each observation was an average of 10 hills. Ten hills were taken randomly from each plot and total numbers of effective tillers hill ${ }^{-1}$ were recorded. Filled and unfilled grains per panicle were counted and averaged from the panicles of ten hills. 1000 grains were taken from the samples and after sun drying the weight was recorded by using electrical balance. The harvested crops was threshed, cleaned, dried and weighed. Straw and grain yields were adjusted to $14 \%$ moisture content. Before land preparation, initial composite soil sample were collected at $0-15 \mathrm{~cm}$ depth from different spots and prepared for physical and chemical analysis. The experimental site is fairly level, well drained and belongs to Deep Red Brown Terrace Soil under Madhupur Tract (AEZ-28). The physical and chemical characteristics of initial soils are given in the table 1.

Table 1. Soil properties of the experimental site

\begin{tabular}{|c|c|c|c|c|c|c|c|c|c|c|c|}
\hline \multirow[t]{2}{*}{ Location } & \multirow[t]{2}{*}{$\mathrm{pH}$} & OM & $\mathrm{Ca}$ & $\mathrm{Mg}$ & $\mathrm{K}$ & Total N & $\mathrm{P}$ & $\mathrm{S}$ & B & Mn & $\mathrm{Zn}$ \\
\hline & & $\%$ & $\mu \mathrm{g} / \mathrm{g}$ & & $\begin{array}{l}\mathrm{cmol} \\
\mathrm{kg}^{-1}\end{array}$ & $(\%)$ & \multicolumn{5}{|c|}{$\mu \mathrm{g} / \mathrm{g}$ soil } \\
\hline SAU, Dhaka & 6.0 & 1.19 & 09 & 0.23 & 0.12 & 0.045 & 16 & 14.4 & 0.1 & 3.0 & 0.4 \\
\hline Critical level & - & - & 2.0 & 0.5 & 0.12 & 0.12 & 10 & 10 & 0.2 & 1.0 & 0.6 \\
\hline
\end{tabular}


Table 2. Nutrient status of organic manure used in the experiment (oven dry basis)

\begin{tabular}{|ccccccc|}
\hline Sources & \multicolumn{6}{c|}{ Nutrient content } \\
\cline { 2 - 7 } & $\mathrm{C}(\%)$ & $\mathrm{N}(\%)$ & $\mathrm{P}(\%)$ & $\mathrm{K}(\%)$ & $\mathrm{S}(\%)$ & $\mathrm{C}: \mathrm{N}$ \\
\hline Cow dung & 36 & 1.20 & 1.00 & 0.75 & 0.21 & 30 \\
Green manure & 46 & 2.00 & 0.26 & 1.56 & 0.24 & 23 \\
\hline
\end{tabular}

Plant nutrient analysis: Grain and straw samples were collected from each plot and were dried for $48 \mathrm{hrs}$ in oven at $65 \pm 5^{\circ} \mathrm{C}$. These dried samples were partitioned into grain and husk. Finally ground samples were passed though $0.5 \mathrm{~mm}$ mesh sieve and were used for chemical determination of $\mathrm{N}, \mathrm{P}$ and $\mathrm{K}$ concentration as described by Jackson (1973). The significance of the differences among pairs of treatment means was estimated by the least significant difference (LSD) test at 5\% level of probability (Gomez and Gomez, 1984). The mean comparisons of the treatment were evaluated by DMRT (Duncan's Multiple Range Test).

\section{RESULTS AND DISCUSSION}

Yield: The highest grain $\left(5.9 \mathrm{t} \mathrm{ha}^{-1}\right)$ and straw $\left(8.59 \mathrm{t} \mathrm{ha}^{-1}\right)$ yields were obtained from treatment $\mathrm{T}_{5}\left(70 \% \mathrm{NPKS}+4 \mathrm{tha}^{-1} \mathrm{DH}\right)$ and $\mathrm{T}_{3}\left(80 \% \mathrm{NPKS}+4 \mathrm{tha}^{-1} \mathrm{CD}\right)$, respectively and the lowest grain yield $\left(3.63 \mathrm{t} \mathrm{ha}^{-1}\right)$ and straw yields $\left(4.66 \mathrm{t} \mathrm{ha}^{-1}\right)$ were observed in control plots (Table 3). Grain yield was positively correlated with the number of effective tiller, panicle length and filled grains panicle ${ }^{-1}$ and 1000 grain weight. The grain and straw yields due to different treatments ranked in order of $\mathrm{T}_{5}>$ $\mathrm{T}_{2}>\mathrm{T}_{3}>\mathrm{T}_{4}>\mathrm{T}_{1}>\mathrm{T}_{7}>\mathrm{T}_{6}>\mathrm{T}_{8}>\mathrm{T}_{10}>\mathrm{T}_{9}>\mathrm{T}_{0}$ and $\mathrm{T}_{3}>\mathrm{T}_{1}>\mathrm{T}_{2}>\mathrm{T}_{4}>\mathrm{T}_{5}>\mathrm{T}_{7}>\mathrm{T}_{6}$ $>\mathrm{T}_{8}>\mathrm{T}_{10}>\mathrm{T}_{9}>\mathrm{T}_{0}$ respectively. Hoque et al. (2001) reported significantly increased grain and straw yields due to application of organic manure and chemical fertilizers. This is also in agreement with the findings of Laxminarayan (2000), Dwivedi and Thakur (2000) and Rajni Rani et al. (2001).

Nitrogen content in grain and straw: Nitrogen contents in rice grain and straw ranged from 1.14 to $1.28 \%$ and 0.710 to $0.862 \%$, respectively. The highest $\mathrm{N}$ contents $(1.28 \%)$ in rice grain and $(0.862 \%)$ in rice straw was observed in the treatment $\mathrm{T}_{1}\left(\mathrm{~N}_{120} \mathrm{P}_{15} \mathrm{~K}_{45} \mathrm{~S}_{20} \mathrm{~kg} \mathrm{ha}^{-1}\right)$ due to application of recommended doses of chemical fertilizers and the lowest $\mathrm{N}$ content in grain $(1.14 \%)$ and in straw $(0.710 \%)$ was noted in $\mathrm{T}_{0}$ (control) treatment (Table 4). The effect of $\mathrm{T}_{5}$ treatment was more pronounced in increasing the $\mathrm{N}$ content in rice grain and straw of BRRI dhan 40 as compared to cow dung. The results revealed that $\mathrm{N}$ content in rice grain was higher than straw. Significant increase in N contents in rice grain and straw due to the application of organic manure and fertilizers have been reported by many investigators (Azim, 1999; Hoque, 1999). 
Table 3. Effects of INM on the grain and straw yields of T. Aman rice (BRRI dhan40)

\begin{tabular}{lcccc}
\hline Treatments & \multicolumn{2}{c}{ Grain } & \multicolumn{2}{c}{ Straw } \\
\cline { 2 - 5 } & $\begin{array}{c}\text { Yield } \\
\left(\mathrm{t} \mathrm{ha}^{-1}\right)\end{array}$ & $\begin{array}{c}\text { Increase over } \\
\text { control }(\%)\end{array}$ & $\begin{array}{c}\text { Yield } \\
\left(\mathrm{t} \mathrm{ha}^{-1}\right)\end{array}$ & $\begin{array}{c}\text { Increase over } \\
\text { control }(\%)\end{array}$ \\
\hline $\mathrm{T}_{0}=$ Control & $3.63 \mathrm{~h}$ & - & $4.66 \mathrm{e}$ & - \\
$\mathrm{T}_{1}=\mathrm{N}_{120} \mathrm{P}_{15} \mathrm{~K}_{45} \mathrm{~S}_{20} \mathrm{~kg} \mathrm{ha}^{-1}(\mathrm{RDF})$ & $5.62 \mathrm{~d}$ & 75.7 & $8.08 \mathrm{ab}$ & 65.5 \\
$\mathrm{~T}_{2}=80 \% \mathrm{NPKS}+2 \mathrm{t} \mathrm{ha}^{-1} \mathrm{DH}$ & $5.85 \mathrm{ab}$ & 84.4 & $8.00 \mathrm{abc}$ & 75.6 \\
$\mathrm{~T}_{3}=80 \% \mathrm{NPKS}+4 \mathrm{t} \mathrm{ha}^{-1} \mathrm{CD}$ & $5.82 \mathrm{~b}$ & 83.3 & $8.59 \mathrm{a}$ & 87.2 \\
$\mathrm{~T}_{4}=80 \% \mathrm{NPKS}+1 \mathrm{t} \mathrm{ha}^{-1} \mathrm{DH}+2 \mathrm{t}$ & $5.75 \mathrm{c}$ & 80.6 & $7.82 \mathrm{bc}$ & \\
$\mathrm{ha}^{-1} \mathrm{CD}$ & & & & 86.5 \\
$\mathrm{~T}_{5}=70 \% \mathrm{NPKS}+4 \mathrm{t} \mathrm{ha}^{-1} \mathrm{DH}$ & $5.90 \mathrm{a}$ & 86.3 & $7.47 \mathrm{bc}$ & 72.5 \\
$\mathrm{~T}_{6}=70 \% \mathrm{NPKS}+8 \mathrm{t} \mathrm{ha}^{-1} \mathrm{CD}$ & $5.18 \mathrm{e}$ & 58.9 & $7.34 \mathrm{c}$ & 65.9 \\
$\mathrm{~T}_{7}=70 \% \mathrm{NPKS}+2 \mathrm{t} \mathrm{ha}^{-1} \mathrm{DH}+4 \mathrm{t}$ & $5.20 \mathrm{e}$ & 59.7 & $7.37 \mathrm{bc}$ & 63.7 \\
$\mathrm{ha}^{-1} \mathrm{CD}$ & & & & 30.2 \\
$\mathrm{~T}_{8}=50 \% \mathrm{NPKS}+6 \mathrm{t} \mathrm{ha}^{-1} \mathrm{DH}$ & $4.53 \mathrm{f}$ & 34.2 & $6.24 \mathrm{~d}$ & 35.2 \\
$\mathrm{~T}_{9}=50 \% \mathrm{NPKS}+12 \mathrm{t} \mathrm{ha}^{-1} \mathrm{CD}$ & $4.45 \mathrm{~g}$ & 31.2 & $6.06 \mathrm{~d}$ & 37.84 \\
$\mathrm{~T}_{10}=50 \% \mathrm{NPKS}+3 \mathrm{t} \mathrm{ha}^{-1} \mathrm{DH}+6 \mathrm{t}$ & $4.52 \mathrm{f}$ & 33.84 & $6.16 \mathrm{~d}$ & \\
$\mathrm{ha}^{-1} \mathrm{CD}$ & & & & - \\
\hline LSD $(\mathrm{P}=0.05)$ & 0.11 & - & 0.13 & - \\
\hline
\end{tabular}

Means having same letter(s) do not differ significantly at 5\% level of probability

$\mathrm{RDF}=$ Recommended Dose of Fertilizer, $\mathrm{N}=$ Nitrogen, $\mathrm{P}=$ Phosphorus, $\mathrm{K}=$ Potassium, $\mathrm{S}=\mathrm{Sulphur}, \mathrm{CD}=$ Cow dung, $\mathrm{DH}=$ Dhaincha, $\pm S E=$ Standard Error of Means, $\mathrm{CD}_{1}=$ Critical Difference

Total $\mathbf{N}$ uptake: The highest total $\mathrm{N}$ uptake $\left(141.9 \mathrm{~kg} \mathrm{ha}^{-1}\right)$ was recorded in the treatment $\mathrm{T}_{1}$ which was statistically identical to treatment $\mathrm{T}_{5}$ (Table 5). This result showed that total $\mathrm{N}$ uptake was high in recommended doses of chemical fertilizer $\mathrm{T}_{1}$ $\left(\mathrm{N}_{120} \mathrm{P}_{15} \mathrm{~K}_{45} \mathrm{~S}_{20} \mathrm{~kg} \mathrm{ha}^{-1}\right)$ followed by combined application of GM with chemical fertilizers (treatment $\mathrm{T}_{5}$ ) . This might be due to (i) increased supply of all essential nutrients directly through organic and inorganic source to crop, (ii) indirectly through checking the losses of nutrient from soil solution and (iii) by increasing in the nutrient use efficiency. Sengar et al. (2000) reported that the $\mathrm{N}$ uptake by rice grain and straw increased significantly with the combined application of organic manure and chemical fertilizers. Duhan et al. (2002); Rahman (2001); Hoque (1999) and Azim (1999) also reported similar results. 
Table 4. Effect of different treatments on N, P, K and S concentration in grain and straw of T. Aman rice (cv. BRRI dhan 40)

\begin{tabular}{|c|c|c|c|c|c|c|c|c|}
\hline \multirow[t]{3}{*}{ Treatments } & \multicolumn{8}{|c|}{ Concentration $(\%)$} \\
\hline & \multicolumn{4}{|c|}{ Grain } & \multicolumn{4}{|c|}{ Straw } \\
\hline & $\mathrm{N}$ & $\mathrm{P}$ & K & $\mathrm{S}$ & $\mathrm{N}$ & $\mathrm{P}$ & K & $\mathrm{S}$ \\
\hline $\mathrm{T}_{0}=$ Control & $1.14 \mathrm{~d}$ & $0.200 \mathrm{~b}$ & $0.258 \mathrm{~b}$ & $0.090 \mathrm{c}$ & $0.710 \mathrm{c}$ & $0.051 \mathrm{~d}$ & $1.573 \mathrm{de}$ & $0.055 \mathrm{c}$ \\
\hline $\mathrm{T}_{1}=\mathrm{N}_{120} \mathrm{P}_{15} \mathrm{~K}_{45} \mathrm{~S}_{20}(\mathrm{RDF})$ & $1.280 \mathrm{a}$ & $0.280 \mathrm{a}$ & $0.324 \mathrm{a}$ & $0.114 \mathrm{ab}$ & $0.862 \mathrm{a}$ & $0.065 \mathrm{bc}$ & $2.120 \mathrm{ab}$ & $0.073 \mathrm{ab}$ \\
\hline $\begin{array}{l}\mathrm{T}_{2}=80 \% \mathrm{NPKS}+2 \mathrm{t} \mathrm{ha}^{-1} \\
\text { DH }\end{array}$ & $1.209 b c$ & $0.292 \mathrm{a}$ & $0.336 \mathrm{a}$ & $0.114 \mathrm{ab}$ & $0.770 \mathrm{bc}$ & $0.081 \mathrm{a}$ & $2.152 \mathrm{ab}$ & $0.083 a$ \\
\hline $\begin{array}{l}\mathrm{T}_{3}=80 \% \mathrm{NPKS}+4 \mathrm{t} \mathrm{ha}^{-1} \\
\mathrm{CD}\end{array}$ & $1.150 \mathrm{~cd}$ & $0.273 \mathrm{a}$ & $0.317 \mathrm{ab}$ & $0.094 \mathrm{c}$ & $0.787 \mathrm{~b}$ & $0.076 \mathrm{a}$ & $1.960 \mathrm{abc}$ & $0.070 \mathrm{~b}$ \\
\hline $\begin{array}{l}\mathrm{T}_{4}=80 \% \mathrm{NPKS}+1 \mathrm{tha}^{-1} \mathrm{DH} \\
+2 \mathrm{tha}^{-1} \mathrm{CD}\end{array}$ & $1.146 \mathrm{~d}$ & $0.280 \mathrm{a}$ & $0.330 \mathrm{a}$ & $0.102 \mathrm{~b}$ & $0.768 \mathrm{bc}$ & $0.058 \mathrm{c}$ & $2.052 \mathrm{ab}$ & $0.078 \mathrm{a}$ \\
\hline $\mathrm{T}_{5}=70 \% \mathrm{NPKS}+4 \mathrm{tha}^{-1} \mathrm{DH}$ & $1.260 \mathrm{ab}$ & $0.289 \mathrm{a}$ & $0.341 \mathrm{a}$ & $0.116 \mathrm{a}$ & $0.778 \mathrm{~b}$ & $0.056 \mathrm{c}$ & $2.250 \mathrm{a}$ & $0.086 \mathrm{a}$ \\
\hline $\mathrm{T}_{6}=70 \% \mathrm{NPKS}+8 \mathrm{tha}^{-1} \mathrm{CD}$ & $1.164 \mathrm{~cd}$ & $0.285 \mathrm{a}$ & $0.315 \mathrm{ab}$ & $0.096 \mathrm{bc}$ & $0.750 \mathrm{bc}$ & $0.079 \mathrm{ab}$ & $1.910 \mathrm{bc}$ & $0.071 \mathrm{~b}$ \\
\hline $\begin{array}{l}\mathrm{T}_{7}=70 \% \mathrm{NPKS}+2 \mathrm{tha}^{-1} \\
\mathrm{DH}+4 \mathrm{tha}^{-1} \mathrm{CD}\end{array}$ & $1.182 \mathrm{~cd}$ & $0.282 \mathrm{a}$ & $0.323 \mathrm{a}$ & $0.099 \mathrm{bc}$ & $0.761 b c$ & $0.063 \mathrm{bc}$ & $1.385 \mathrm{e}$ & $0.077 \mathrm{ab}$ \\
\hline $\begin{array}{l}\mathrm{T}_{8}=50 \% \mathrm{NPKS}+6 \mathrm{tha}^{-} \\
{ }^{{ }^{1} \mathrm{DH}}\end{array}$ & $1.157 \mathrm{~cd}$ & $0.279 \mathrm{a}$ & $0.312 \mathrm{ab}$ & $0.113 \mathrm{ab}$ & $0.779 \mathrm{~b}$ & $0.077 \mathrm{~b}$ & $2.150 \mathrm{ab}$ & $0.079 \mathrm{ab}$ \\
\hline $\begin{array}{l}\mathrm{T}_{9}=50 \% \text { NPKS }+12 \text { tha } \\
{ }^{1} \mathrm{CD}\end{array}$ & $1.160 \mathrm{~cd}$ & $0.255 \mathrm{ab}$ & $0.298 \mathrm{ab}$ & $0.106 \mathrm{~b}$ & $0.754 \mathrm{bc}$ & $0.079 \mathrm{ab}$ & $1.722 \mathrm{~cd}$ & $0.070 \mathrm{~b}$ \\
\hline $\begin{array}{l}\mathrm{T}_{10}=50 \% \text { NPKS }+3 \mathrm{tha} \\
{ }^{1} \mathrm{DH}+6 \mathrm{tha}^{-1} \mathrm{CD}\end{array}$ & $1.150 \mathrm{~cd}$ & $0.251 \mathrm{ab}$ & $0.290 \mathrm{ab}$ & $0.096 \mathrm{bc}$ & $0.752 b c$ & $0.055 \mathrm{c}$ & $1.690 \mathrm{cde}$ & $0.073 \mathrm{ab}$ \\
\hline$\pm \mathrm{SE}$ & 1.15 & 0.28 & 0.23 & 0.001 & 0.002 & 0.056 & 1.18 & 0.03 \\
\hline $\mathrm{CD}_{1}(\mathrm{P}=0.05)$ & 0.33 & 0.81 & 0.45 & 0.004 & 0.004 & 0.045 & 0.37 & 0.08 \\
\hline
\end{tabular}

Means having same letter(s) do not differ significantly at 5\% level of probability

$\mathrm{RDF}=$ Recommended Dose of Fertilizer, $\mathrm{N}=$ Nitrogen, $\mathrm{P}=$ Phosphorus, $\mathrm{K}=$ Potassium, $\mathrm{S}=$ Sulphur, $\mathrm{CD}=$ Cowdung, $\mathrm{DH}=$ Dhaincha, $\pm S E=$ Standard Error of Means, $\mathrm{CD}_{1}=$ Critical Difference

Phosphorus content in grain and straw: Phosphorus content in rice grain and straw ranged from 0.200 to $0.292 \%$ and 0.051 to $0.081 \%$, respectively. The highest $\mathrm{P}$ content $(0.292 \%)$ in grain and $(0.081 \%)$ in rice straw was observed in the treatment $\mathrm{T}_{2}\left(0 \%\right.$ NPKS $\left.+2 \mathrm{tha}^{-1} \mathrm{DH}\right)$ and the lowest $\mathrm{P}$ concentration was noted in the treatment $\mathrm{T}_{0}$ (Table 4$)$. The next highest $\mathrm{P}$ content in rice grain and straw was found in the treatment $\mathrm{T}_{5}\left(70 \% \mathrm{NPKS}+4 \mathrm{t} \mathrm{ha}^{-1} \mathrm{DH}\right)$. Lower grain P content was observed in those treatment received NPKS fertilizers with CD compared to 100\% NPKS and NPKS fertilizers with GM. Verma (1992) reported that incorporation of organic manure significantly increased the concentration of $\mathrm{P}$ in rice grain and straw yields of rice. 
Table 5. Nitrogen and Phosphorus uptake by grain and straw of T. Aman rice (cv. BRRI dhan 40)

\begin{tabular}{|c|c|c|c|c|c|c|}
\hline \multirow[t]{2}{*}{ Treatments } & \multicolumn{2}{|c|}{$\mathrm{N}$ uptake $\left(\mathrm{kg} \mathrm{ha}^{-1}\right)$} & \multirow{2}{*}{$\begin{array}{c}\text { Total N } \\
\text { uptake }(\mathrm{kg} \\
\left.\mathrm{ha}^{-1}\right)\end{array}$} & \multicolumn{2}{|c|}{ P uptake $\left(\mathrm{kg} \mathrm{ha}^{-1}\right)$} & \multirow{2}{*}{$\begin{array}{l}\text { Total P } \\
\text { uptake } \\
\left(\mathrm{kg} \mathrm{ha}^{-1}\right)\end{array}$} \\
\hline & Grain & Straw & & Grain & Straw & \\
\hline $\mathrm{T}_{0}=$ Control & $41.38 \mathrm{e}$ & $33.08 \mathrm{e}$ & $74.46 f$ & $7.26 \mathrm{f}$ & $2.38 \mathrm{~g}$ & $9.64 \mathrm{i}$ \\
\hline $\mathrm{T}_{1}=\mathrm{N}_{120} \mathrm{P}_{15} \mathrm{~K}_{45} \mathrm{~S}_{20}(\mathrm{RDF})$ & $71.94 \mathrm{a}$ & $69.65 \mathrm{a}$ & $141.59 \mathrm{a}$ & $15.74 \mathrm{~b}$ & $5.25 \mathrm{c}$ & $20.99 \mathrm{~cd}$ \\
\hline $\mathrm{T}_{2}=80 \% \mathrm{NPKS}+2 \mathrm{t} \mathrm{ha}^{-1} \mathrm{DH}$ & $70.73 \mathrm{a}$ & $61.40 \mathrm{~b}$ & $132.13 \mathrm{bc}$ & $17.05 \mathrm{a}$ & $6.32 \mathrm{a}$ & $23.40 \mathrm{a}$ \\
\hline $\mathrm{T}_{3}=80 \% \mathrm{NPKS}+4 \mathrm{tha}^{-1} \mathrm{CD}$ & $70.30 \mathrm{~b}$ & $67.60 \mathrm{a}$ & $138.9 \mathrm{ab}$ & $15.89 \mathrm{~b}$ & $6.53 \mathrm{a}$ & $22.42 \mathrm{~b}$ \\
\hline $\begin{array}{l}\mathrm{T}_{4}=80 \% \mathrm{NPKS}+1 \mathrm{tha}^{-1} \mathrm{DH}+ \\
2 \mathrm{t} \mathrm{ha}^{-1} \mathrm{CD}\end{array}$ & $65.90 \mathrm{~b}$ & $60.06 \mathrm{bc}$ & $125.96 \mathrm{c}$ & $16.10 \mathrm{~b}$ & $4.53 \mathrm{~d}$ & $20.63 \mathrm{~d}$ \\
\hline $\mathrm{T}_{5}=70 \% \mathrm{NPKS}+4 \mathrm{tha}^{-1} \mathrm{DH}$ & $69.85 \mathrm{a}$ & $58.10 \mathrm{bc}$ & $132.44 \mathrm{bc}$ & $17.08 \mathrm{a}$ & $4.16 \mathrm{e}$ & $21.15 \mathrm{c}$ \\
\hline $\mathrm{T}_{6}=70 \% \mathrm{NPKS}+8 \mathrm{tha}^{-1} \mathrm{CD}$ & $60.30 \mathrm{c}$ & $55.05 \mathrm{c}$ & $115.35 \mathrm{~d}$ & $14.76 \mathrm{c}$ & $5.95 \mathrm{~b}$ & $20.71 \mathrm{~cd}$ \\
\hline $\begin{array}{l}\mathrm{T}_{7}=70 \% \mathrm{NPKS}+2 \mathrm{tha}^{-1} \mathrm{DH} \\
+4 \mathrm{tha}^{-1} \mathrm{CD}\end{array}$ & $61.46 \mathrm{c}$ & $56.09 \mathrm{c}$ & $117.55 \mathrm{~d}$ & $14.67 \mathrm{c}$ & $4.64 \mathrm{~d}$ & $19.31 \mathrm{e}$ \\
\hline $\mathrm{T}_{8}=50 \% \mathrm{NPKS}+6 \mathrm{tha}^{-1} \mathrm{DH}$ & $52.41 \mathrm{~d}$ & $48.61 \mathrm{~d}$ & $101.02 \mathrm{e}$ & $12.64 d$ & $4.80 \mathrm{~d}$ & $17.44 \mathrm{f}$ \\
\hline $\mathrm{T}_{9}=50 \% \mathrm{NPKS}+12 \mathrm{tha}^{-1} \mathrm{CD}$ & $51.62 \mathrm{~d}$ & $45.69 \mathrm{~d}$ & $97.31 \mathrm{e}$ & $11.35 \mathrm{e}$ & $4.79 \mathrm{~d}$ & $16.14 \mathrm{~g}$ \\
\hline $\begin{array}{l}\mathrm{T}_{10}=50 \% \mathrm{NPKS}+3 \mathrm{tha}^{-1} \mathrm{DH} \\
+6 \mathrm{tha}^{-1} \mathrm{CD}\end{array}$ & $51.98 \mathrm{~d}$ & $46.32 \mathrm{~d}$ & $98.3 \mathrm{e}$ & $11.35 \mathrm{e}$ & $3.39 \mathrm{f}$ & $14.74 \mathrm{~h}$ \\
\hline$\pm \mathrm{SE}$ & 4.18 & 2.3 & 1.23 & 0.001 & 0.002 & 0.456 \\
\hline $\mathrm{CD}_{1}(\mathrm{P}=0.05)$ & 3.37 & 2.8 & 3.45 & 0.004 & 0.004 & 1.45 \\
\hline
\end{tabular}

Means having same letter(s) do not differ significantly at $5 \%$ level of probability

RDF= Recommended Dose of Fertilizer, $\mathrm{N}=$ Nitrogen, $\mathrm{P}=$ Phosphorus, $\mathrm{K}=$ Potassium, $\mathrm{S}=\mathrm{Sulphur}, \mathrm{CD}=$ Cow dung, $\mathrm{DH}=$ Dhaincha, $\pm S E=$ Standard Error of Means, $\mathrm{CD}_{1}=$ Critical Difference

Total P uptake: The total $\mathrm{P}$ uptake ranged from 9.64 to $23.40 \mathrm{~kg} \mathrm{ha}^{-1}$. The highest total P uptake $\left(23.40 \mathrm{~kg} \mathrm{ha}^{-1}\right)$ was recorded in the treatment $\mathrm{T}_{2}\left(80 \% \mathrm{NPKS}+2 \mathrm{tha}^{-1}\right.$ $\mathrm{DH})$, and the lowest value $\left(9.64 \mathrm{~kg} \mathrm{ha}^{-1}\right)$ was observed in the treatment $\mathrm{T}_{0}$ (Table 5). This results shows that total $\mathrm{P}$ uptake by grain and straw were significantly due to combined application of GM with chemical fertilizers. Sengar et al. (2000) reported that the $\mathrm{P}$ uptake by rice grain and straw increased significantly with the combined application of organic manure and chemical fertilizers.

Potassium content in grain and straw: The highest $\mathrm{K}$ content $(0.341 \%)$ in grain and (2.25\%) straw was observed in the treatment $\mathrm{T}_{5}\left(70 \% \mathrm{NPKS}+4 \mathrm{t} \mathrm{ha}^{-1} \mathrm{DH}\right)$ that was significantly higher than the rest of the treatments and the lowest $\mathrm{K}$ concentration was noted in the treatment $\mathrm{T}_{0}$ (Table 4). From the results it was observed that the $\mathrm{K}$ content in rice straw was higher than grain in all the treatments $\mathrm{K}$ contents both in grain and straw of rice increased due to combined application of organic manure and chemical fertilizers (Islam, 1997; Khan et al., 1998). 
Table 6. Potassium and S uptake by grain and straw of T. Aman rice (BRRI dhan40)

\begin{tabular}{|c|c|c|c|c|c|c|}
\hline \multirow[t]{2}{*}{ Treatments } & \multicolumn{2}{|c|}{$\mathrm{K}$ uptake $\left(\mathrm{kg} \mathrm{ha}^{-1}\right)$} & \multirow{2}{*}{$\begin{array}{l}\text { Total K uptake } \\
\left(\mathrm{kg} \mathrm{ha}^{-1}\right)\end{array}$} & \multicolumn{2}{|c|}{ S uptake $\left(\mathrm{kg} \mathrm{ha}^{-1}\right)$} & \multirow{2}{*}{$\begin{array}{c}\text { Total S } \\
\text { uptake }(\mathrm{kg} \\
\left.\mathrm{ha}^{-1}\right)\end{array}$} \\
\hline & Grain & Straw & & Grain & Straw & \\
\hline $\mathrm{T}_{0}=$ Control & $9.37 \mathrm{f}$ & $73.32 \mathrm{e}$ & $82.69 \mathrm{~d}$ & $3.27 \mathrm{~h}$ & $2.57 \mathrm{~h}$ & $5.84 \mathrm{~g}$ \\
\hline $\mathrm{T}_{1}=\mathrm{N}_{120} \mathrm{P}_{15} \mathrm{~K}_{45} \mathrm{~S}_{20}(\mathrm{RDF})$ & $18.21 \mathrm{c}$ & $171.29 \mathrm{a}$ & $189.5 \mathrm{a}$ & $6.41 \mathrm{~b}$ & $5.90 \mathrm{bc}$ & $12.31 \mathrm{~b}$ \\
\hline $\mathrm{T}_{2}=80 \% \mathrm{NPKS}+2 \mathrm{tha}^{-1} \mathrm{DH}$ & $19.66 \mathrm{ab}$ & $172.15 \mathrm{a}$ & $191.81 \mathrm{a}$ & $6.67 \mathrm{ab}$ & $6.64 \mathrm{a}$ & $13.31 \mathrm{a}$ \\
\hline $\mathrm{T}_{3}=80 \% \mathrm{NPKS}+4 \mathrm{tha}^{-1} \mathrm{CD}$ & $18.45 \mathrm{bc}$ & $168.38 \mathrm{a}$ & $186.83 \mathrm{a}$ & $5.47 \mathrm{~d}$ & $6.01 \mathrm{bc}$ & $11.48 \mathrm{c}$ \\
\hline $\begin{array}{l}\mathrm{T}_{4}=80 \% \mathrm{NPKS}+1 \mathrm{tha}^{-1} \mathrm{DH}+2 \mathrm{t} \\
\mathrm{ha}^{-1} \mathrm{CD}\end{array}$ & $18.98 \mathrm{abc}$ & $160.46 \mathrm{ab}$ & $179.44 \mathrm{ab}$ & $5.87 \mathrm{c}$ & $6.10 \mathrm{abc}$ & $11.97 \mathrm{bc}$ \\
\hline $\mathrm{T}_{5}=70 \% \mathrm{NPKS}+4 \mathrm{tha}^{-1} \mathrm{DH}$ & $20.12 \mathrm{a}$ & $168.01 \mathrm{a}$ & $188.13 \mathrm{a}$ & $6.84 \mathrm{a}$ & $6.42 \mathrm{ab}$ & $13.26 \mathrm{a}$ \\
\hline $\mathrm{T}_{6}=70 \% \mathrm{NPKS}+8 \mathrm{tha}^{-1} \mathrm{CD}$ & $16.32 \mathrm{~d}$ & $148.51 \mathrm{abc}$ & $164.83 \mathrm{ab}$ & $4.97 \mathrm{e}$ & $5.21 \mathrm{de}$ & $10.18 \mathrm{e}$ \\
\hline $\begin{array}{l}\mathrm{T}_{7}=70 \% \mathrm{NPKS}+2 \mathrm{tha}^{-1} \mathrm{DH}+4 \mathrm{t} \\
\mathrm{ha}^{-1} \mathrm{CD}\end{array}$ & $16.80 \mathrm{~d}$ & $123.55 \mathrm{~cd}$ & $140.3 \mathrm{bc}$ & $5.15 \mathrm{e}$ & $5.67 \mathrm{~cd}$ & $10.82 \mathrm{~d}$ \\
\hline $\mathrm{T}_{8}=50 \% \mathrm{NPKS}+6 \mathrm{tha}^{-1} \mathrm{DH}$ & $14.13 \mathrm{e}$ & $132.13 \mathrm{bcd}$ & $146.27 \mathrm{~b}$ & $5.12 \mathrm{e}$ & $4.93 \mathrm{ef}$ & $10.05 \mathrm{e}$ \\
\hline $\mathrm{T}_{9}=50 \% \mathrm{NPKS}+12 \mathrm{tha}^{-1} \mathrm{CD}$ & $13.26 \mathrm{e}$ & $104.35 \mathrm{~d}$ & $117.61 \mathrm{c}$ & $4.72 \mathrm{f}$ & $4.24 \mathrm{~g}$ & $8.96 \mathrm{f}$ \\
\hline $\begin{array}{l}\mathrm{T}_{10}=50 \% \mathrm{NPKS}+3 \mathrm{tha}^{-1} \mathrm{DH}+6 \mathrm{t} \\
\mathrm{ha}^{-1} \mathrm{CD}\end{array}$ & $13.11 \mathrm{e}$ & $104.11 \mathrm{~d}$ & $117.22 \mathrm{c}$ & $4.34 \mathrm{~g}$ & $4.50 \mathrm{fg}$ & $8.84 \mathrm{f}$ \\
\hline$\pm_{\mathrm{SE}}$ & 0.002 & 0.003 & 2.15 & 0.44 & 2.6 & 0.002 \\
\hline $\mathrm{CD}_{1}(\mathrm{P}=0.05)$ & 0.003 & 0.005 & 4.54 & 0.35 & 6.67 & 0.003 \\
\hline
\end{tabular}

Means having same letter(s) do not differ significantly at $5 \%$ level of probability

$\mathrm{RDF}=$ Recommended Dose of Fertilizer, $\mathrm{N}=$ Nitrogen, $\mathrm{P}=$ Phosphorus, $\mathrm{K}=$ Potassium, $\mathrm{S}=$ Sulphur, $\mathrm{CD}=$ Cow dung, $\mathrm{DH}=$ Dhaincha, $\pm S E=$ Standard Error of Means, $\mathrm{CD}_{1}=$ Critical Difference

Total K uptake: The highest total K uptake $\left(191.81 \mathrm{~kg} \mathrm{ha}^{-1}\right)$ was recorded in the treatment $\mathrm{T}_{2}\left(80 \% \mathrm{NPKS}+2 \mathrm{t} \mathrm{ha}^{-1} \mathrm{DH}\right)$, and the lowest value $\left(82.69 \mathrm{~kg} \mathrm{ha}^{-1}\right)$ was observed in the treatment $\mathrm{T}_{0}$ (Table 6). This results shows that total $\mathrm{K}$ uptake were influenced more due to combined application of GM with chemical fertilizers. Sengar et al. (2000) reported that the $\mathrm{K}$ uptake by rice grain and straw increased significantly with the combined application of organic manure and chemical fertilizers.

Sulphur content in grain and straw: The highest $S$ content $(0.116 \%)$ in grain and $(0.086 \%)$ in rice straw was observed in the treatment $\mathrm{T}_{5}\left(70 \% \mathrm{NPKS}+4 \mathrm{t} \mathrm{ha}^{-1} \mathrm{DH}\right)$ that was significantly higher than the rest of the treatments and the lowest $\mathrm{S}$ concentration was noted in the treatment $\mathrm{T}_{0}$ (Table 4). Azim (1999) and Hoque (1999) reported that application of $\mathrm{S}$ from manure and fertilizers increased $\mathrm{S}$ content both in grain and straw. Ali et al. (2009) also reported the similar results.

Total S uptake: The highest total S uptake $\left(13.31 \mathrm{~kg} \mathrm{ha}^{-1}\right)$ was recorded in the treatment $\mathrm{T}_{2}\left(80 \% \mathrm{NPKS}+2 \mathrm{t} \mathrm{ha}^{-1} \mathrm{DH}\right)$ and the lowest value $\left(5.84 \mathrm{~s} \mathrm{~kg} \mathrm{ha} \mathrm{kg}^{-1}\right)$ was observed in the treatment $\mathrm{T}_{0}$ (Table 6). Azim (1999) and Hoque (1999) recorded the higher uptake of $\mathrm{S}$ with the application of manure and fertilizers in combinations.

Post harvest soil properties: Application of organic manure and chemical fertilizers resulted in considerable influence on the properties of the post-harvest soils (Table 
7). In some treatments the $\mathrm{pH}$ value slightly decreased as compared to the initial value (6.0). The highest organic matter content $(1.42 \%)$ was found in $\mathrm{T}_{5}(70 \%$ NPKS $\left.+4 \mathrm{tha}^{-1} \mathrm{DH}\right)$ treatment which was also increased compared to the initial value. Organic manuring increased the organic matter content, total $\mathrm{N}$, available $\mathrm{P}$, exchangeable $\mathrm{K}$ and available $\mathrm{S}$ in the post harvest soils. Hoque et al. (2001), Mathew and Nair (1997), and Azim (1999) reported that, combined application of organic manure and chemical fertilizer can increased the organic matter content, total $\mathrm{N}$, available $\mathrm{P}$, exchangeable $\mathrm{K}$ and available $\mathrm{S}$ in the post-harvest soils.

Table 7. Effect of INM on post-harvest soils properties

\begin{tabular}{|c|c|c|c|c|c|c|}
\hline Treatments & Soil $\mathrm{pH}$ & $\begin{array}{c}\text { Organic } \\
\text { matter }(\%)\end{array}$ & $\begin{array}{c}\text { Total N } \\
(\%)\end{array}$ & $\begin{array}{l}\text { Available } \\
\mathrm{P}(\mathrm{ppm})\end{array}$ & $\begin{array}{c}\text { Exchange } \\
\text { able K } \\
(\text { me \%) }\end{array}$ & $\begin{array}{l}\text { Available } S \\
\quad(\mathrm{ppm})\end{array}$ \\
\hline $\mathrm{T}_{0}=$ Control & $5.92 \mathrm{ab}$ & $1.28 \mathrm{c}$ & $0.065 \mathrm{a}$ & $17.48 \mathrm{j}$ & $0.099 \mathrm{e}$ & $11.02 \mathrm{c}$ \\
\hline $\mathrm{T}_{1}=\mathrm{N}_{120} \mathrm{P}_{15} \mathrm{~K}_{45} \mathrm{~S}_{20} \mathrm{~kg} \mathrm{ha}^{-1}$ (RDF) & $5.98 \mathrm{a}$ & $1.39 \mathrm{ab}$ & $0.095 \mathrm{a}$ & $19.45 d$ & $0.135 \mathrm{c}$ & $18.168 \mathrm{a}$ \\
\hline $\mathrm{T}_{2}=80 \% \mathrm{NPKS}+2 \mathrm{tha}^{-1} \mathrm{DH}$ & $5.80 \mathrm{bc}$ & $1.34 \mathrm{~b}$ & $0.089 \mathrm{a}$ & $19.09 \mathrm{f}$ & $0.147 \mathrm{ab}$ & $19.665 \mathrm{a}$ \\
\hline $\mathrm{T}_{3}=80 \% \mathrm{NPKS}+4 \mathrm{tha}^{-1} \mathrm{CD}$ & $6.00 \mathrm{a}$ & $1.36 \mathrm{ab}$ & $0.090 \mathrm{a}$ & $19.31 \mathrm{e}$ & $0.134 \mathrm{c}$ & $16.450 \mathrm{ab}$ \\
\hline $\begin{array}{l}\mathrm{T}_{4}=80 \% \mathrm{NPKS}+1 \mathrm{tha}^{-1} \mathrm{DH}+2 \mathrm{t} \\
\mathrm{ha}^{-1} \mathrm{CD}\end{array}$ & $5.78 \mathrm{c}$ & $1.36 \mathrm{ab}$ & $0.092 \mathrm{a}$ & $18.56 \mathrm{i}$ & $0.148 \mathrm{ab}$ & $17.752 \mathrm{a}$ \\
\hline $\mathrm{T}_{5}=70 \% \mathrm{NPKS}+4 \mathrm{t} \mathrm{ha}^{-1} \mathrm{DH}$ & $5.86 \mathrm{bc}$ & $1.42 \mathrm{a}$ & $0.097 \mathrm{a}$ & $19.95 \mathrm{c}$ & $0.156 \mathrm{a}$ & $18.280 \mathrm{a}$ \\
\hline $\mathrm{T}_{6}=70 \% \mathrm{NPKS}+8 \mathrm{tha}^{-1} \mathrm{CD}$ & $5.82 b c$ & $1.38 \mathrm{ab}$ & $0.088 \mathrm{a}$ & $23.98 \mathrm{a}$ & $0.150 \mathrm{a}$ & $16.225 \mathrm{ab}$ \\
\hline $\begin{array}{l}\mathrm{T}_{7}=70 \% \mathrm{NPKS}+2 \mathrm{tha}^{-1} \mathrm{DH}+4 \mathrm{t} \\
\mathrm{ha}^{-1} \mathrm{CD}\end{array}$ & $5.88 \mathrm{abc}$ & $1.36 \mathrm{ab}$ & $0.089 \mathrm{a}$ & $20.12 b$ & $0.140 \mathrm{a}$ & $17.558 \mathrm{a}$ \\
\hline $\mathrm{T}_{8}=50 \% \mathrm{NPKS}+6 \mathrm{tha}^{-1} \mathrm{DH}$ & $5.90 \mathrm{abc}$ & $1.38 \mathrm{ab}$ & $0.090 \mathrm{a}$ & $18.96 \mathrm{~g}$ & $0.142 b c$ & $17.770 \mathrm{a}$ \\
\hline $\mathrm{T}_{9}=50 \% \mathrm{NPKS}+12 \mathrm{tha}^{-1} \mathrm{CD}$ & $5.84 b c$ & $1.38 \mathrm{ab}$ & $0.082 \mathrm{a}$ & $18.80 \mathrm{~h}$ & $0.112 d$ & $12.520 \mathrm{bc}$ \\
\hline $\begin{array}{l}\mathrm{T}_{10}=50 \% \mathrm{NPKS}+3 \mathrm{tha}^{-1} \mathrm{DH}+6 \\
\mathrm{t} \mathrm{ha}^{-1} \mathrm{CD}\end{array}$ & $6.00 \mathrm{a}$ & $1.35 \mathrm{~b}$ & $0.079 \mathrm{a}$ & $18.66 \mathrm{i}$ & $0.141 b c$ & $16.665 \mathrm{ab}$ \\
\hline$\pm \mathrm{SE}$ & 0.001 & 0.001 & 0.001 & 0.44 & 0.58 & 0.55 \\
\hline $\mathrm{CD}_{1}(\mathrm{P}=0.05)$ & 0.002 & 0.003 & 0.003 & 1.77 & 2.37 & 1.44 \\
\hline
\end{tabular}

Means having same letter(s) do not differ significantly at $5 \%$ level of probability

$\mathrm{RDF}=$ Recommended Dose of Fertilizer, $\mathrm{N}=$ Nitrogen, $\mathrm{P}=$ Phosphorus, $\mathrm{K}=$ Potassium, $\mathrm{S}=$ Sulphur, $\mathrm{CD}=$ Cowdung, $\mathrm{DH}=$ Dhaincha, $\pm S E=$ Standard Error of Means, $\mathrm{CD}_{1}=$ Critical Difference

\section{CONCLUSION}

From the present study it may be concluded that rice variety BRRI dhan 40 responded better to the nutrient supplied from the organic manure in producing grain and straw yields. The study clearly demonstrates that the benefit of using dhaincha as GM can reduce the N, P, K and S fertilizers for T. Aman rice, giving good economic yield and also slightly increased the soil organic matter content particularly when the fertilizers were applied on soil test basis (STB). This is the one year result. Regular practice of INM may be able to increase different soil qualities including soil organic matter content. BRRI dhan 40 can be cultivated profitably in the Tejgoan silt loam 
soil by using combined application of $70 \%$ NPKS fertilizers with $4 \mathrm{t} \mathrm{ha}^{-1} \mathrm{GM}$. The overall findings of this study indicate that the combined use of fertilizer and manure in T. Aman rice should be encouraged for maintaining rice yield, quality and soil fertility.

\section{REFERENCES}

Ali, M.E., Islam, M.R. and Jahiruddin, M. 2009 . Effect of integrated use of organic manures with chemical fertilizers in the rice-rice cropping system and its impact on soil health. Bangladesh Journal of Agricultural research, 34(1): 81-90

Ali, M.M., Shaheed, S.M. and Kubota, D. 1997.Soil degradation during the period of 19671995 in Bangladesh II. Selected chemical characters. Journal of Soil Science \& Plant Nutrition, 43: 890Anonymous.2010.http://www.slidefinder.net/t/life_your_soil/introductionsoil_micr obiology/ 1116901. environmental soils.cas.psu.edu.

Azim, S.M.A. 1999. Effect of sulphur, zinc and boron supplied from manure and fertilizers on BRRI 29. M.S. Thesis, Dept. Soil Sci., (Jan-June, 1999. Sem.), BAU, Mymensingh

BARC, 2012. Fertilizer Recommendation Guide. Bangladesh Agricultural Research Council, Farmgate, Dhaka-1215, $274 \mathrm{p}$

BBS (Bangladesh Bureau of Statistics). 2010. Statistical Year book of Bangladesh. Stat. Div. Min. Planning, Dhaka, Bangladesh

BRRI (Bangladesh Rice Research Institute). 2006. Annual Report for 2006. Bangladesh Rice Res. Inst. Joydebpur, Gazipur

Bhuiyan, N.I., Shaha, A.L. and Panaullah, G.M. 1991. Effect of NPK fertilizer on the grain yield of transplanted rice and soil fertility. Longterm study. Bangladesh Journal of Soil Science, 22 (1\&2): 41-50

Cassman, K.G., Datta, de.S.K., OIK. D.C., Alcantra, J., Sason, M., Descalsota, J. and Dizon. 1995. Yield decline and nitrogen economy of long term experiment on continuous irrigated rice system in the tropics. pp. 181-122. In: R. Lal and Stewart, B. A. (eds.) Soil Management: Experimental basis for sustainability and environmental quality. lewis Publisher, London

Dwivedi, D.K. and Thakur, S.S. 2000. Effect of organic and inorganic fertilizers levels on productivity of rice (Oriza sativa) crop. Indian Journal of Agricultural Research, 45(3): $568-574$

Duhan, B.S. and Singh, M. 2002. Effect of green manuring and nitrogen on the yield and nutrient uptake of micronutrient by rice. Journal of the Indian Society of Soil Science, 50(2): $178-180$

Hoque, M.A. 1999. Response of BRRI dhan 29 to sulpher, zinc and boron supplied from manure and fertilizers, M.S. Thesis, Dept. Soil Sci. (January-june, 1999, Sem.) BAU, Mymensingh

Hedge, D.M. 1998. Long-term sustainability of productivity in system in sub-humid ecosystem through integrated nutrient supply. Indian Journal of Agricultural Research, 43(2): 189-198 
Islam, A.1992. Review of Soil Fertility Research in Bangladesh. Proceeding of the InterCongress Conference of Commission IV. December 1-3, 1992, Dhaka. Improving Soil Management for Intensive Cropping in the Tropic and Sub-tropics. BARC. Pub. No. 37: $1-18$

Jackson, M.L. 1973. Soil Chemical Analysis. Constable and Co. Ltd. London, First Print

Karim, Z., Miah, M.M.U. and Razia, S. 1994. Fertilizer in the national economy and sustainable environmental development. Asia Pacific Journal of Environment 1: 48-67

Khan, S.K., Mohanty, S.K. and Chalam, A.B. 1998. Integrated management of organic manure and and nitrogen fertilizer for rice. Journal of the Indian Society of Soil Science, 34(3): 693-697

Laxminarayan, K. 2000. Effect of integrated use of organic and inorganic manures on yield of low land pady. Journal of Hill Research, 13(2): 125-127

Meelu, O.P. and Singh, Y. 1992. Integrated use of fertilizers and organic manure for higher returns.Prog.Fmg. Punjab Agric. Univ. 27: 3-4

Mondal, 2000. Bangladesh Agricultural Research Institute. Project report, Bio-slurry Management and Its Effect on Soil Fertilizer and Crop Production, $5 \mathrm{p}$

Nambiar, K.K.M. 1997. Long-term fertility effects on wheat productivity. In: wheat for the Non-traditional Warm areas, Saunders D.A. ed., CIMMYT .pp 516-521

Rahman, M.A. 2001. Integrated use of fertilizer and manure for crop production in wheat-rice and rice-rice cropping patterns. Ph.D. Thesis. Dept. Soil Sci., Bangladesh AgriI. Univ., Mymensingh

Rajni, R., Srivastava, O.P., and Rani. R. 2001. Effect of integration of organics with fertilizer $\mathrm{N}$ on rice and $\mathrm{N}$ uptake. Fertilizer News, 46(9): 63-65

Sengar, S.S., Wade, L.J., Baghel, S.S., Singh, R.K. and Singh, G. 2000. Effect of nutrient management on rice (Oryza sativa) in rain fed low land of Southeast Madhya Pradesh. Indian Journal of Agricultural Research, 45(2): 315-322

Shaheen. A., Fatma, M., Rizk, A. and Singer, S.M. 2007. Growing onion plants without chemical fertilization. Research Journal of Agriculture and Biological Sciences, 3 (2): 95-104

Singh, R., Singh, S. and Prasad, K.2001. Effect of fertilizer, FYM and row spacing on transplanted rice. Crop Research, (Hisar). 22(2): 295-296

Verma, T.S. and R.M. Bhagat. 1992. Impact of rice straw management practices on yield, nitrogen uptake and soil properties in a wheat -rice rotation in northern India. Fertilizer Research, 33:97-106 